Document downloaded from:

http://hdl.handle.net/10251/121779

This paper must be cited as:

Sanchez Saez, F.; Sánchez Galdón, Al.; Villanueva López, JF.; Carlos Alberola, S.; Martorell Alsina, SS. (2018). Uncertainty analysis of a large break loss of coolant accident in a pressurized water reactor using non-parametric methods. Reliability Engineering \& System Safety. 174:19-28. https://doi.org/10.1016/j.ress.2018.02.005

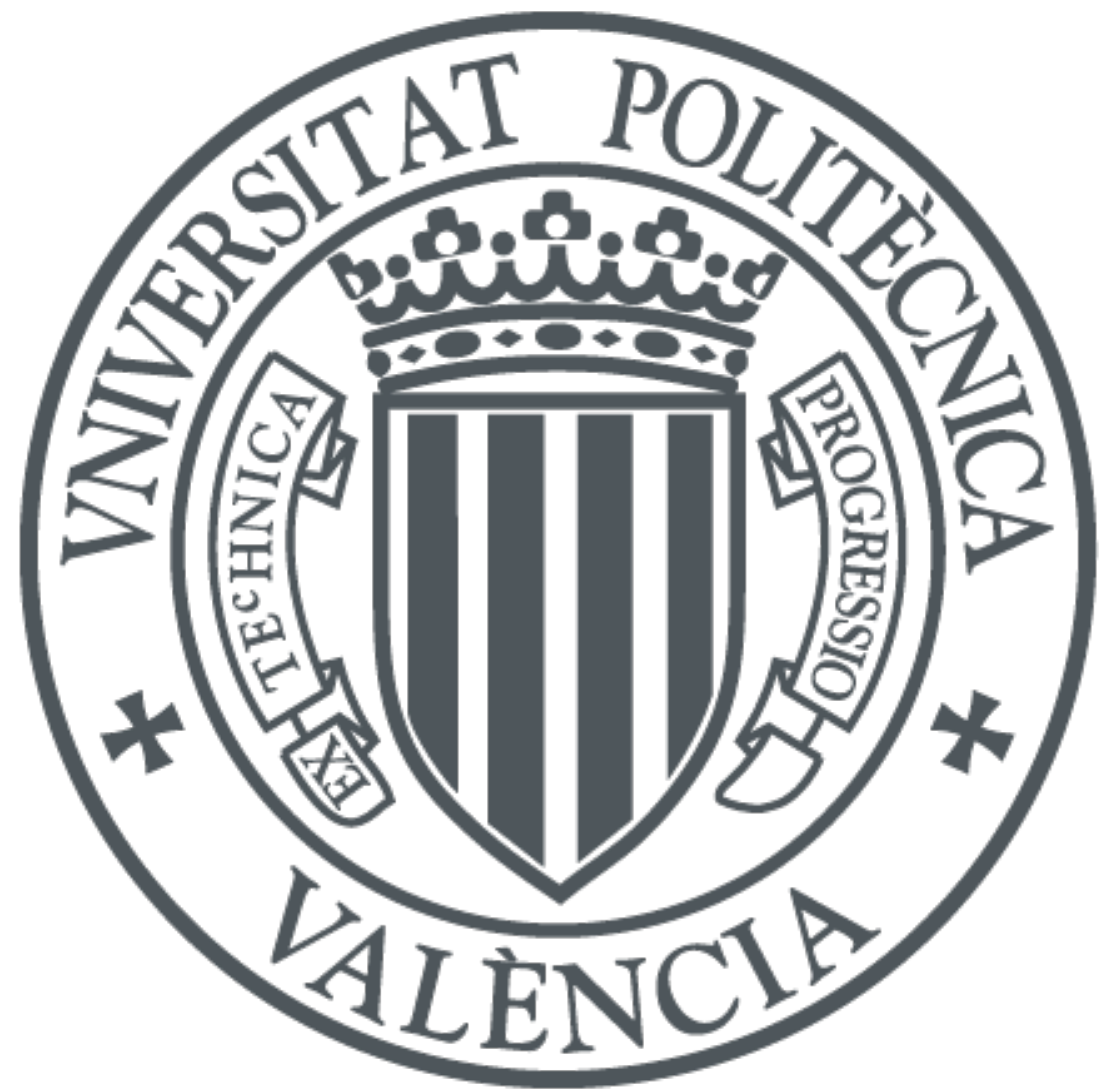

The final publication is available at

https://doi.org/10.1016/j.ress.2018.02.005

Copyright Elsevier

Additional Information 


\title{
Uncertainty analysis of a Large Break Loss of Coolant Accident in a Pressurized Water Reactor using non-parametric methods
}

\author{
F. Sanchez-Saez, A.I. Sánchez, J.F. Villanueva, S. Carlos, S. Martorell \\ Universitat Politècnica de València, Camí de Vera s/n, 46021, Valencia - Spain \\ frasansa@etsii.upv.es: aisanche@eio.upv.es; jovillo0@iqn.upv.es; scarlos@iqn.upv.es; \\ smartore@iqn.upv.es
}

\begin{abstract}
The safety analysis of nuclear power plant is moving towards a realistic approach in which the simulations performed using best estimate computer codes must be accompanied by an uncertainty analysis, known as the Best Estimate Plus Uncertainties approach. The most popular statistical method used in these analyses is the Wilks' method, which is based on the principle of order statistics for determining a certain coverage of the Figures-of-Merit with an appropriate degree of confidence. However, there exist other statistical techniques that could provide similar or even better results. This paper explores the performance of alternative nonparametric methods as compared to the Wilks' method of obtaining such Figure-of-Merits tolerance intervals. Three methods are investigated, i.e. Hutson and Beran-Hall methods and a bootstrap method. All the techniques have been used to perform the uncertainty analysis of a Large-Break Loss of Coolant Accident. The Figure-of-Merit of interest in this application is the maximum value reached by the Peaking Clad Temperature. In order to analyze the results obtained by the different methods, four performance metrics are proposed to measure the coverage, dispersion, conservativeness, and robustness of the tolerance intervals.
\end{abstract}

Keyword: Uncertainty; Best-Estimate plus uncertainty; deterministic safety analysis; Large Break Loss of Coolant Accident; Wilks' method; order statistics, non-parametric methods 


\section{NOTATION}

$\alpha \quad$ Acceleration parameter in $\mathrm{BC} \alpha$ method

$B(n, p)$ Binomial distribution of parameters $n$ and $p$

$C_{j} \quad$ Coverage of the sample $j$

$C C$ Conservativeness

$F(\cdot) \quad$ Cumulative distribution function (cdf)

$\hat{F}^{-1} \quad$ Inverse of the empirical cdf

$f(\cdot) \quad$ Probability density function (pdf)

$I[c]$ Indicator function. This function is equal to 1 if $c$ is true and 0 if $c$ is false

$I_{x}(a, b)$ Incomplete beta function of parameters $x, a$ and $b$

$Z \quad$ Reference distribution sample size

$n \quad$ Sample size for estimating TL

$N \quad$ Number of samples (repetitions)

$p \quad$ Coverage of the tolerance interval

$U_{n^{\prime} p: n} \quad$ Dirichlet process

$X_{i: n} \quad i^{\text {th }}$ order statistics from a sample of size $n$ of independent and identically distributed random variables

$z_{0} \quad$ Bias correction parameter in $\mathrm{BC}_{\alpha}[\gamma]$ method

$z_{\gamma} \quad \mathrm{Z}$ score from the standard normal distribution

$\gamma \quad$ Confidence level of the tolerance interval

$\xi_{p} \quad p$ percentile

$\Phi(\cdot) \quad$ Standard normal cdf

\section{ACRONYMS}

$\mathrm{BC}_{\alpha}[\gamma] \quad$ Bias Corrected Accelerated bootstrap method

$\mathrm{BE}$

Best Estimate

BEPU Best Estimate Plus Uncertainty

CD Coverage standard deviation

CHF Critical Heat Flux

$\mathrm{CM} \quad$ Coverage Mean

CV Coverage Coefficient of Variation

DSA Deterministic Safety Analysis

ECCS Emergency core cooling systems

FOM Figure of Merit

FOS First Order Statistic

GAMA Working Group on Accident Management and Analysis

IAEA International Atomic Energy Agency

LBLOCA Large-Break Loss of Coolant Accident

LPIS Low-Pressure Injection System

NPP Nuclear Power Plant

OS Order Statistic

PCT Peaking Clad Temperature

PIRT Process Identification and Ranking Tables

PWR Pressurized Water Reactor

$\mathrm{SV}_{\text {ref }} \quad$ Reference Safety Value

TH Thermal Hydraulic

TL Tolerance limit

UA Uncertainty analysis

TRACE TRAC-RELAP Advanced Computational Engine

SNAP Symbolic Nuclear Analysis Package 


\section{INTRODUCTION}

The International Atomic Energy Agency's guidance (IAEA) on the use of deterministic safety analysis (DSA) for the design and licensing of nuclear power plants (NPPs) "Deterministic Safety Analysis for Nuclear Power Plants Specific Safety Guide, Specific Safety Guide No. SSG-2" [1] (hereinafter referred to as SSG-2) addresses four options for DSA applications. Due to the importance of taking the current understanding of physical phenomena into account, and thanks to the availability of reliable tools for more realistic safety analyses without compromising plant safety, many countries have chosen Option 3.

Option 3 involves the use of best-estimate codes and data together with an evaluation of the uncertainties, the so-called Best Estimated Plus Uncertainty (BEPU) methodologies. Table 1 shows the different options addressed in the SSG-2 guide.

Table 1: SSG-2 DSA options

\begin{tabular}{lccc}
\hline \multicolumn{1}{c}{ Option } & Computer code & Availability of systems & Initial and boundary conditions \\
\hline 1. Conservative & Conservative & Conservative assumptions & Conservative input data \\
\hline 2. Combined & Best Estimate & Conservative assumptions & Conservative input data \\
\hline 3. Best estimate & Best Estimate & Conservative assumptions & $\begin{array}{c}\text { Realistic input data plus uncertainty; } \\
\text { partly most unfavourable } \\
\text { conditions }^{\mathrm{a}}\end{array}$ \\
\hline 4. Risk-informed & Best Estimate & $\begin{array}{c}\text { Derived from probabilistic } \\
\text { safety analysis }\end{array}$ & $\begin{array}{c}\text { Best Realistic input data } \\
\text { with uncertainties }^{\mathrm{a}}\end{array}$ \\
\hline
\end{tabular}

\footnotetext{
${ }^{a}$ Realistic input data are used only if the uncertainties or their probabilistic distributions are known. For those parameters whose uncertainties are not quantifiable with a high level of confidence, conservative values should be used.
}

The IAEA Safety Report Series N ${ }^{\circ} .23$ "Accident Analysis for Nuclear Power Plants" [2] recommends a sensitivity and uncertainty analysis if Best Estimate (BE) codes are used in the licensing analysis. A comprehensive overview of uncertainty methods can be found in the IAEA Safety Report Series N ${ }^{\circ} .52$ "Best Estimate Safety Analysis for Nuclear Power Plants: Uncertainty Evaluation", issued in 2008 [3]. References [4,5,6,7] deal with the evolution of BEPU analysis and describe some of the most frequently used techniques. Some of these techniques have been developed by International programs which have discussed the BEPU approaches in order to address the issue of the capabilities of best-estimate computational tools and uncertainty analysis.

This is the case, for example, of the BEMUSE, promoted by the Working Group on Accident Management and Analysis (GAMA) of the OECD. These discussions have led to the development of BEPU approaches insofar as they have been accepted for performing deterministic safety analysis by the regulatory authorities. The scope of BEMUSE Phase V, in which fourteen participants from twelve organizations and ten countries participated, is the uncertainty analysis of a Large Break Loss-Of-Coolant-Accident (LBLOCA) in a Pressurized Water Reactor. The results and the main lessons learned from this BEMUSE program are presented in reference [8].

In a BEPU design-basis accident it is normally assumed that the uncertainty in the safety outputs [i.e., the figures of merit (FOMs) involved in the acceptance criteria of the analysis] derives from the uncertainties in the input parameters (initial and boundary conditions) and those arising from the computational model [4]. 
These FOMs are usually extreme values (minima, maxima) of safety variables during the transient, such as Peak Clad Temperature (PCT), Critical Heat Flux (CHF), etc. Current BEPU methodologies mainly rely on a probabilistic description of the uncertainty and on the use of statistical techniques to estimate it $[9,10,11,12,13]$. In this framework, the uncertainty of a FOM can be identified with its probability distribution.

Most BEPU approaches accepted by the regulatory authorities are based on the propagation of input uncertainties and make use of methods based on Wilks' formula, which is based on the principle of order statistics for determining a certain coverage of the Figures-Of-Merit (FOM) with a certain degree of confidence. The German Technical Safety Organisation (TSO) Gesellschaft für Anlagen- und Reaktorsicherheit (GRS) was the first to introduce Wilks' tolerance limits in uncertainty analyses with TH codes, so that this type of analysis is renowned as the GRS method [9]. This method determines the number of code runs needed to obtain a sample of outputs, i.e. FOMs, which are required to verify compliance with acceptance criteria. In accordance with current regulatory practice a $95 \%$ coverage with a $95 \%$ confidence level is required. So, if a one-side FOM tolerance interval is applied based on the use of the First Order Statistics (FOS) with a 95/95 coverage/confidence level a sample size of $n=59$ runs is required.

This paper focuses on the deterministic safety analysis of a Large-Break Loss of Coolant Accident (LBLOCA) scenario in a PWR NPP based on a BEPU approach and the use of order statistics according to the current practice for the formulation, propagation, and analysis of uncertainties. In addition, the paper introduces alternative non-parametric methods to the traditional first order statistics based on Wilks' formulae. The results of the alternative methods are compared with those of the traditional method based on appropriate performance metrics also proposed in this paper. The study specifically focuses on the analysis of the uncertainty associated with the maximum of the PCT (Peak Cladding Temperature) as the FOM.

\section{OVERVIEW OF THE BEPU APPROACH}

Figure 1 outlines a typical procedure used in BEPU approaches [14], which consist of the following twelve steps:

1. Selection of the accident scenario. Reactor system and transient selection.

2. Selection of the safety criteria linked to the accident scenario under study and the FOM involved in the acceptance criteria.

3. Identification and ranking of relevant physical phenomena based on the safety criteria.

4. Selection of the appropriate uncertain TH (Thermal Hydraulic) parameters to represent those phenomena.

5. Identification of relevant safety-related functions and systems involved in the accident scenario.

6. Identify relevant trains and components of the safety-related functions and systems developing their possible redundancies.

7. Development of the TH computer model of the accident scenario, e.g. developing an input for the TRACE integrated into the SNAP platform $[15,16,17]$.

8. Allocation of PDF (Probability Density Functions) for each selected uncertain TH parameter.

9. Establishing conservative assumptions on the availability of trains/components of safety systems.

10. Random sampling of the selected uncertain TH parameters according to PDF. Sample size (n) will depend on the particular statistical method and the acceptance criteria 
adopted to verify compliance with safety criteria. Perform $n$ computer runs to obtain the value of the FOM for each run.

11. Processing the results of the multiple computer runs (n) to estimate either the probability distribution of the FOM, or rather some descriptor of this distribution, such as for example a percentile of the FOM, or a tolerance limit of FOM using OS, etc.

12. Verify compliance of acceptance criteria for each FOM depending on the particular statistical method and acceptance criteria adopted.

Despite SSG 2 recommends the development of uncertainty analysis based on the use of OS (normally first order to produce FOM with 95/95 tolerance limit (TL)), several alternatives can be explored integrating not only uncertainty but also sensitivity analyses to produce more realistic and accurate results while maintaining an acceptable computational cost. Some of these alternatives are based on the use of non-parametric methods.

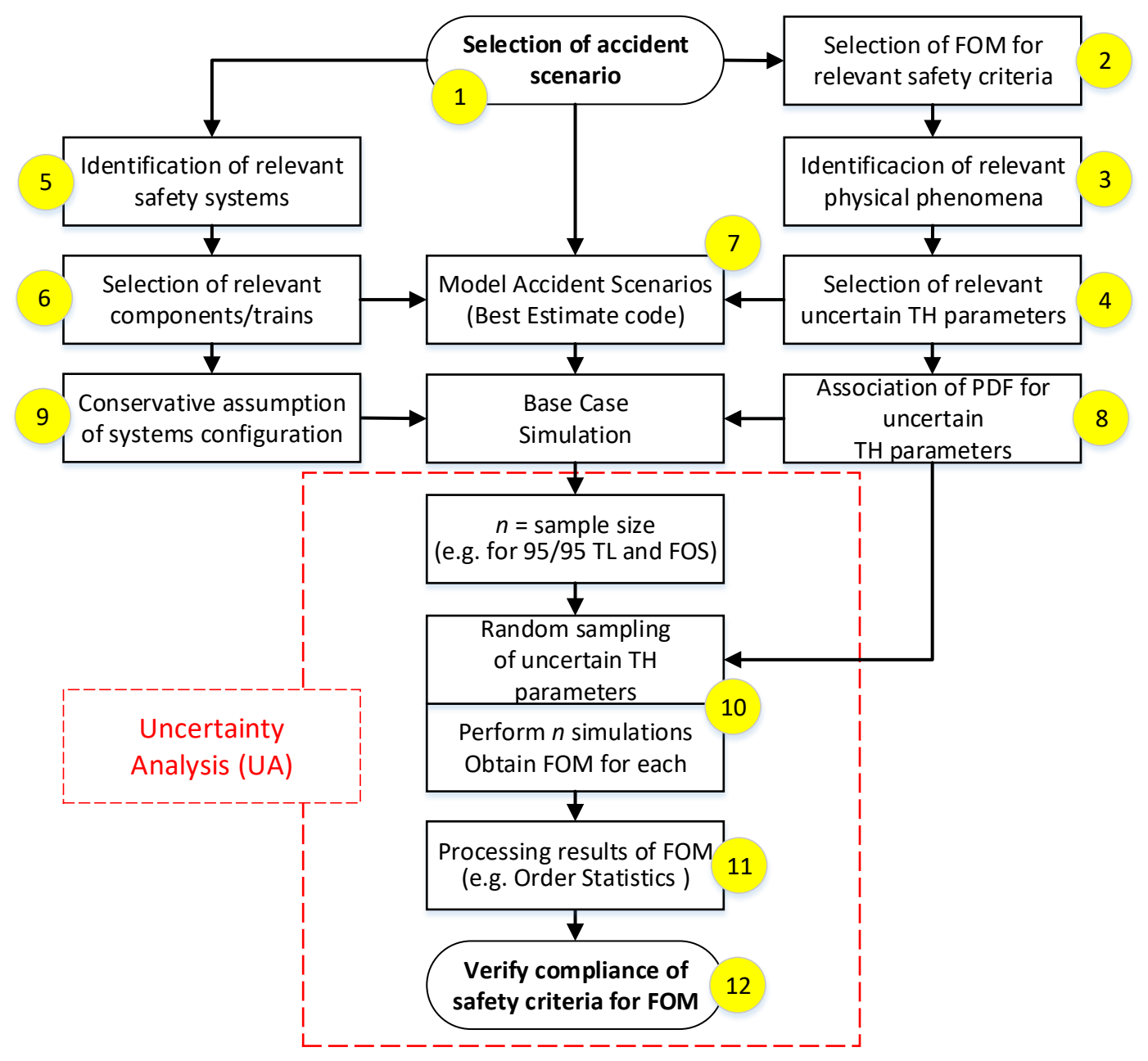

Figure 1. Overview of a typical BEPU approach.

\section{UNCERTAINTY ANALYSIS BASED ON TOLERANCE INTERVALS}

Since BEPU analyses have been performed by the Nuclear Regulatory Commission (NRC) [18], several uncertainty approaches have been proposed and applied in analyses approved by the nuclear industry [19]. The Wilks' method [20, 21] is the most popular non-parametric statistical method used in thermo-hydraulic codes uncertainty analysis [9]. It is based on the idea of order statistics and requires a minimum number of thermal-hydraulic code simulations 
in order to infer a certain tolerance limit of a given output from the code simulations. The use of the Wilks' method and first order statistics mostly leads to conservative results. Another alternative to solve this problem consists of using higher order statistics, which can avoid overconservative results because of outliers.

Alternative non-parametric approaches have also been proposed in the literature with the aim of reducing the level of conservatism. These approaches are aimed at meeting a one-sided confidence limit for a percentile, which is also a one-side tolerance limit. Thus, Beran \& Hall [22] and Hutson [23] proposed two approaches to estimate a non-parametric confidence interval for percentiles. The bootstrap method is also a data-based method that can be used to estimate a confidence interval for a parameter of interest, e.g. percentiles. These methods are outlined in the following subsections.

\subsection{Wilks' method}

The main advantage of using First Order Statistics based on Wilks' formulae to derive the 95/95 TL is that it provides a conservative result with only a few computer code runs. This way, the computational cost is kept reasonable, since the simulation of the evolution of the plant transient for each sample of inputs using complex TH (Thermal Hydraulic) NPP models is expensive in terms of computational cost. However, FOS often provides excessively conservative results. Several authors have explored the advantage of using order statistics of higher levels, which reduce the conservatism at the expense of a higher computational cost [24, 25]. Others propose the use of sensitivity and uncertainty analysis in an integrated manner within the framework of the BEPU approach [2, 8]. Non-parametric methods have been proposed with a similar purpose, such as the Bootstrap method introduced in reference [26].

Wilks' method uses the Order Statistics (OS) as tolerance limits. Let $X$ be a (continuous) random variable with a cumulative distribution function (cdf), and $X_{1}, X_{2}, \ldots, X_{n}$ be a simple random sample of size $n$ of $X$. Let $X_{1: n} \leq X_{2: n} \leq \ldots \leq X_{r: n} \leq \ldots \leq X_{n: n}$ be the order statistics from the sample. According to Wilks' method $[14,15]$ the smallest number $n$ of code runs to be performed to obtain the $r$-th order one-sided tolerance interval, or tolerance limit, of a given FOM is given by the following inequality:

$1-\sum_{k=n-r+1}^{n}\left(\begin{array}{l}n \\ k\end{array}\right) p^{k}(1-p)^{n-k} \geq \gamma$

where $n$ is the sample size, $p$ is the coverage probability and $\gamma$ is the confidence level of the one-sided tolerance interval. It is common practice in BEPU applications, for example to LBLOCA scenarios, to use the 95/95 tolerance limit $(p / \gamma)$ according to current regulatory practice. When first order statistics (FOS) (i.e. $r=1$ ) is employed, it imposes the use of a sample size of 59 sets of TH input parameters randomly selected from their $p d f$ and performing 59 code runs, one for each set.

However, in most cases using FOS leads to conservative results. Applying the Wilks' formula to higher order statistics usually produces more accurate FOM tolerance limits but increases the computational burden, as more runs are needed. For example, if $r$ is 3 and $p / \gamma$ are both $0.95 / 0.95$, the minimum number of code runs needed to satisfy such criteria is $n=124$. Table 2 shows the minimum number of code runs from the $1^{\text {st }}$ to $4^{\text {th }}$ order statistics necessary to determine a 95/95 TL. 
Table 2: Minimum number of code runs

\begin{tabular}{cc}
\hline Order Wilks' formula & $\begin{array}{c}\text { Minimum number of } \\
\text { code runs (n) }\end{array}$ \\
\hline $\mathbf{1}$ & 59 \\
$\mathbf{2}$ & 93 \\
$\mathbf{3}$ & 124 \\
$\mathbf{4}$ & 153 \\
\hline
\end{tabular}

\subsection{Beran and Hall's simple linear interpolation}

Beran and Hall's simple linear interpolation provides an interesting approach to obtaining nonparametric confidence intervals for population percentiles based on a random sample of size $n$. They used a convex combination of sample percentiles to develop linearly interpolated confidence intervals with interpolation weights based on binomial distribution.

Let $n(p, \gamma)$ be the minimum sample size required for the calculation of a $p / \gamma$ one-sided tolerance limit obtained from Eq. (1) for $r=1$, i.e. FOS. For a sample of size $n^{\prime} \geq n(p, \gamma)$ let $X_{s: n}$ be the one-sided upper tolerance limit obtained using the Wilks' method with a confidence level $\gamma_{1} \geq$ $\gamma$. Additionally, the confidence level of $X_{s-1: n}$ is $\gamma_{2}<\gamma$. An estimation of $X\left(s^{*}\right)$ can be obtained by linear interpolation between $\left(\gamma_{2}, X_{s-1: n}\right)$ and $\left(\gamma_{1}, X_{s: n}\right)$. Thus, for a confidence level $\gamma$ :

$$
\begin{aligned}
& \gamma_{1}=\operatorname{Pr}(\mathrm{B}(n, p) \leq s-1)=\operatorname{Pr}\left(\xi_{p} \in\left[-\infty, X_{\text {s:n }}\right]\right) \geq \gamma \\
& \gamma_{2}=\operatorname{Pr}(\mathrm{B}(n, p) \leq s-2)=\operatorname{Pr}\left(\xi_{p} \in\left[-\infty, X_{\text {s-1:n } n}\right]\right)<\gamma
\end{aligned}
$$

Where $\mathrm{B}$ is the binomial distribution of parameters $n$ and $p$ and $\xi_{p}$ is the $p$ percentile. Therefore, $p / \gamma$ upper tolerance limit for any distribution is a $\gamma$ upper confidence limit for the $p$ percentile of the distribution.

Beran and Hall showed that using linear interpolation the value of the one-sided upper tolerance limit corresponding to a coverage $p$ and nominal confidence $\gamma$ is given by:

$$
\lambda_{2} \cdot X_{s: n}+\left(1-\lambda_{2}\right) \cdot X_{s-1: n}
$$

so that

$$
\lambda_{2}=\frac{\gamma-\operatorname{Pr}(B(n, p) \leq s-2)}{\operatorname{Pr}(B(n, p)=s-1)}
$$

\subsection{Hutson fractional statistics}

Hutson [23] proposes an approach to constructing non-parametric confidence intervals for percentiles based on fractional statistics. Thus, if $U_{n^{\prime} p: n}$ is a Dirichlet process with $p \in[0,1]$ indexed by $n^{\prime} p$ where $n^{\prime}=n+1$ then $U_{n} p: n$ is the fractional uniform order statistic and $X_{n} p: n$ is the fractional order statistic for the distribution $F_{x}($.$) . Stigler [27] outlines the properties of$ $U_{n^{\prime} p: n}$ : 
a) $U_{n^{\prime} p: n}$ has a beta distribution with parameters $n^{\prime} p$ and $n^{\prime}(1-p)$,

b) $E\left[U_{n^{\prime} p: n}\right]=p$ and $\operatorname{Var}\left[U_{n^{\prime} p: n}\right]=p(1-p) /(n+2)$.

c) The random variable $U_{n^{\prime} p: n}$ has the same distribution as the uniform order statistic $U_{i: n}$ at $n^{\prime} p=i$, $i=1,2, . ., n$.

d) The random variable $F^{-1}\left(U_{n^{\prime} p: n}\right)$ has the same distribution as the order statistic $X_{i: n}$ at $n^{\prime} p=i$, $i=1,2, \ldots, n$ being $F^{-1}$ the inverse distribution function.

The estimator of the $p$-percentile based on the fractional order statistic is defined as:

$\xi_{p}=X_{n^{\prime} p: n}$

In general, $X_{n^{\prime} p: n}$ cannot be calculated from the sample since $n^{\prime} p$ need not be an integer. In reference [28] is shown that the distribution of the fractional order statistic is well approximated by the distribution of linear combinations of order statistics, under moderate regularity conditions. So, the distribution of the fractional order statistic $\xi_{p}=X_{n^{\prime} p: n}$ can be approximated by the distribution of the linear interpolation estimator of the percentile function as

$\xi_{p}=(1-\varepsilon) \cdot X_{\left\lfloor n^{\prime} p\right\rfloor: n}+\varepsilon \cdot X_{\left\lfloor n^{\prime} p\right\rfloor+1: n}$

where $\varepsilon=n^{\prime} p-\left\lfloor n^{\prime} p\right\rfloor$, being \lfloor\rfloor the floor function.

Hutson obtains the $\gamma$ nonparametric confidence interval for the $p$ percentile as $\left[-\infty, \xi_{p 1}\right]$ where $p_{1}$ is determined numerically by solving the equation

$I_{p}\left[n^{\prime} p_{1}, n^{\prime}\left(1-p_{1}\right)\right]=1-\gamma$

where $I_{p}$ is the incomplete beta function and $\xi_{p 1}$ is given by Eq. (7).

\subsection{Bootstrap method}

The bootstrap method is a data-based method in which statistical inference is obtained by data sampling. The bootstrap is a computationally intensive, nonparametric technique for assessing the accuracy of a parameter estimator which requires very few assumptions or analysis $[27,28$, $29,30]$. The goal of bootstrap confidence interval theory is to calculate confidence limits for a parameter of interest $\theta$ from the bootstrap distribution of $\hat{\theta}$.

It is possible to obtain confidence bounds of percentiles from the bootstrap sampling distribution. Different methods have been proposed in the literature to obtain confidence bounds from the bootstrap, for example, percentile method, bias-corrected accelerated method, approximated bootstrap confidence interval, bootstrap-t method or a combination of EnsembleBased Sensitivity Analysis and the Bootstrap method [26].

In this paper, the bias-corrected accelerated $\left(\mathrm{BC}_{\alpha}[\gamma]\right)$ method is used for calculating one-side upper 95/95 TL. This method has a number of advantages: 1) it corrects for bias and skewness in the distribution of bootstrap estimates, 2) no estimates of the standard deviation are required and 3) minimum coverage error. 
The $\mathrm{BC}_{\alpha}[\gamma]$ procedure [24] is a method of setting approximate confidence intervals for $\theta$ from the percentiles of the bootstrap histogram. Suppose $\theta$ is a parameter of interest, in this case, the p-percentile $\left(\xi_{p}\right), \hat{\theta}(\boldsymbol{x})$ is an estimate of $\theta$ based on the observed data $\boldsymbol{x}$ and $\hat{\theta}^{*}=\hat{\theta}\left(x^{*}\right)$ is a bootstrap replication of $\hat{\theta}$ obtained by resampling $x^{*}$. Let $\hat{F}(c)$ be the empirical cumulative distribution function of $\mathrm{B}$ bootstrap replications $\hat{\theta}^{*}(b)$ with $\mathrm{b}=1,2, \ldots, \mathrm{B}$, thus is:

$$
\widehat{F}(c)=\#\left\{\hat{\theta}^{*}(b)<c\right\} / B
$$

The upper confidence interval limit $\hat{\theta}_{B C \alpha}[\gamma]$ of a one-side level $-\gamma \mathrm{BC}_{\alpha}$ interval is defined as a function of $\hat{F}$, empirical $c d f$, and two parameters: The bias correction $z o$, which is related to the proportion of bootstrap estimates that are less than the observed statistic, and the acceleration $\alpha$, proportional to the skewness of the bootstrap distribution. By definition, $\hat{\theta}_{B C \alpha}[\gamma]$ is the upper confidence interval limit, which is given by:

$$
\hat{\theta}_{B C_{\alpha}[\gamma]}=\hat{F}^{-1} \Phi\left(z_{0}+\frac{z_{0}+z_{\gamma}}{1-\alpha\left(z_{0}+z_{\gamma}\right)}\right)
$$

Where $\hat{F}^{-1}$ is the inverse empirical $c d f$ of the bootstrap sampling distribution of $\hat{\theta}(b), z_{\gamma}$ is the critical value of the normal standard distribution for a given $\gamma$ thus is $z_{\gamma}=\Phi^{-1}(\gamma)$, and $\Phi$ is the standard normal $c d f$.

The bias-correction $z_{0}$ is estimated by

$$
z_{0}=\Phi^{-1}(\hat{F}(\hat{\theta}))
$$

And so can be computed directly from the bootstrap distribution. The acceleration factor $\alpha$ can be approximated [28] by:

$$
\alpha=\frac{S K E W \hat{l}_{\theta}(\hat{\theta})}{6}
$$

SKEW $\hat{l}_{\theta}(\hat{\theta})$ being the skewness at parameter value $\theta=\hat{\theta}$ of the score statistic $\hat{l}_{\theta}(\hat{\theta}) . \hat{l}_{\theta}(\hat{\theta})$ is the first derivate of the $\log$-likelihood $l$ for $\theta$, thus $\hat{l}_{\theta}(\hat{\theta})=\frac{\partial}{\partial \theta} \log \left\{f_{\theta}(\hat{\theta})\right\}$, where $f_{\theta}(\hat{\theta})$ is the probability density function, $\hat{\theta}$ is the sample estimate of $\theta$ and the likelihood is taken under bootstrap sampling.

\subsection{Performance metrics}

In nuclear deterministic safety analysis using a BEPU approach, the results of the uncertainty analysis must meet the regulatory requirements. The Wilks' method is the widest uncertainty technique used in this field, although has been proved to be over-conservative in some cases. It therefore seems necessary to find metrics able to evaluate the goodness of the results found by the alternative methods and compare them to the Wilks' method in terms of conservatism. 
In this work, the metrics proposed in reference [31] have been adapted as follows. First, a reference sample of size $Z$, e.g. $Z=1000$, is generated using the $c d f$ of the input parameters, i.e. $\mathrm{Z}$ sets of input values for the BE code are obtained. The BE code is then run for each set of the input values, so that $Z$ simulations of the code output are obtained, which permits us to obtain $Z$ FOM values, i.e. one for each simulation result. Let $y_{i}$ be the $i$-th FOM value $(i=1,2, \ldots, Z)$, which is the result of the $\mathrm{i}$-th BE code simulation.

Next, a size $\mathrm{n}$ is adopted from the set $n=\{59,93,124,153\}$, depending on the OS of interest. For example, $n=59$ is considered to obtain the FOS. The process explained below can be repeated for each $n$ value to cover all the OS of interest.

Then, from the complete set of Z FOM values, $N$ samples of a given size $n$ are extracted, which are analyzed with the different non-parametric methods described in Sections 3.1 to 3.4. The results of these analyses are estimations of 95/95 TL of the FOM according to each method and for each of the $N$ samples of size $n$. From these FOM 95/95 TL results the following performance metrics can be obtained of interest for the comparison of the several nonparametric methods.

On one hand, the coverage reached under each method for each sample $j=1, \ldots, N$ of size $n$ is calculated, which represents the percentage of the reference distribution covered by the $\mathrm{j} 95 / 95$ tolerance limit $\left(\mathrm{TL}_{\mathrm{j}}\right)$ estimated for each $j=1, \ldots, N$. It can be evaluated using the following expression:

$$
\left.C_{j}=\frac{1}{Z}\left(\sum_{i=1}^{Z} I\left[\left(y_{i}\right) \leq T L_{j}\right)\right]\right)
$$

where $y_{i}$ is the $i$-th FOM value, $\mathrm{TL}_{j}$ is the $95 / 95$ tolerance limit estimated with some of the uncertainty methods for each $j$ case, and $\mathrm{I}[x]$ is the indicator function, which equals 1 if $x$ is true and 0 if $x$ is false. With the $C_{j}$, the different descriptive measures are evaluated:

1) Coverage Mean (CM) of the individual coverages as a measure of coverage performance of the method.

2) Coverage Standard Deviation (CD), as a measure of variability of the results.

3) Coefficient of Variation (CV). It is a measure of the robustness of the result, which is evaluated as follows:

$$
C V=\frac{C D}{C M} \cdot 100
$$

On the other hand, it is also possible to quantify the degree of conservativeness (CC) of each method, which represents the number of results out of the total of $N$ samples that are conservative for each method. This can be evaluated as follows:

$$
\left.C C=\frac{1}{N}\left(\sum_{j=1}^{N} I\left[\left(T L_{j}\right) \geq S V_{r e f}\right)\right]\right) \cdot 100
$$

where $S V_{\text {ref }}$ is the reference safety value, which, for the purpose of this study, is the $95 \%$ percentile of the distribution of the FOM, and can be estimated using the reference sample of size $Z=1000 \gg n$. 


\section{CASE STUDY}

The methods introduced in the previous sections are applied to the uncertainty analysis of an LBLOCA in the cold leg of a typical 4-loops PWR-Westinghouse reactor design, whose plant of reference is Zion NPP. When the break occurs the primary system rapidly depressurizes and activates the SCRAM signal due to low pressure. This leads to the safety injection through accumulators followed by actuation of the Low-Pressure Injection System (LPIS) to prevent core uncovery.

\subsection{Safety variables and acceptance criteria}

Following the methodology described in Fig. 1, it is necessary to determine the safety variables and their acceptance criteria to be considered in the study. Several safety criteria were established by USNRC for LOCA accidents in 1974 and last reviewed in 2015 [32]. These criteria can be mainly summarized into three requirements that must be in compliance: the peak cladding temperature, the maximum cladding oxidation of the core, and the maximum of the total amount of hydrogen produced during the transient. The safety variable usually followed in thermo-hydraulic safety analysis is the Peak Cladding Temperature (PCT), with a limiting maximum value of $1477 \mathrm{~K}$. As this criterion was also used in this study, the PCT represents the safety variable of interest and the maximum value of the PCT, i.e. PCTmax is the FOM to be considered in the BEPU analysis.

\subsection{Relevant physical phenomena, thermal hydraulics parameters, and PDF}

Once the accident scenario (LBLOCA), the safety variable of interest (PCT), the corresponding FOM (PCTmax) and the acceptance criterion (PCTmax below $1477 \mathrm{~K}$ ) have been selected, it is necessary to identify and rank the important phenomena and the most important $\mathrm{TH}$ parameters affecting the transient evolution and consequently also the value of the PCTmax reached.

A Process Identification and Ranking Tables (PIRT) process is often used to select the most important phenomena and parameters. Reference [6] contains a comparison of three PIRTs for LBLOCA in 4 loops PWR NPP, corresponding to three studies performed by CSAU [33], AREVA [19] and Westinghouse/EPRI [34]. In this comparison, common phenomena in the three studies are observed, such as heat transfer in single and two-phase flow, rod behavior, etc. However, significant differences were also found in the three studies. The latest studies on BEMUSE [4] and PREMIUM [35] have brought new findings, for example, on the re-flooding phenomena.

In the present study, the relevant phenomena and parameters identified in these PIRT studies and other parameters that have been incorporated into the new modeling capabilities introduced in the BE TRACE code V5.0 Patch 4 using the SNAP suite have been considered. Table 3 shows the $68 \mathrm{TH}$ parameters selected, together with their nominal value and their uncertainty taken from the above references, the latter being introduced through a multiplicative or additive factor that acts as either multiplier or additive factor for the nominal values of the parameters.

The probability density functions that characterize each of these parameters are normal, uniform and lognormal distributions, as shown in Table 3, where Normal distributions are 
truncated in \pm 2 standard deviations. The two lognormal distributions have $\mu=0, \sigma=0.3536$, with a shift to 0.5 and truncated to 2 .

Table 3. Description of uncertain TH input parameters

\begin{tabular}{|c|c|c|c|c|}
\hline Normal Distributed Parameters & $\begin{array}{l}\text { Nominal Value } \\
\text { (units) }\end{array}$ & $\begin{array}{l}\text { Factor } \\
\text { type }\end{array}$ & Mín & Máx \\
\hline Initial thermal power & 3250 (MW) & Mult. & 0.98 & 1.02 \\
\hline Peaking factor & $1.2468(-)$ & Mult. & 0.95 & 1.05 \\
\hline Hot gap size average & $5.4 \mathrm{e}-5(\mathrm{~m})$ & Mult. & 0.8 & 1.2 \\
\hline Hot gap size hot rod & $5.4 \mathrm{e}-5(\mathrm{~m})$ & Mult. & 0.8 & 1.2 \\
\hline Residual power multiplier & $1(-)$ & Mult. & 0.92 & 1.08 \\
\hline $\mathrm{UO}_{2}$ thermal conductivity & $1(-)$ & Mult. & 0.9 & 1.1 \\
\hline $\mathrm{UO}_{2}$ specific heat & $1(-)$ & Mult. & 0.98 & 1.02 \\
\hline Intact loops pump speed multiplier & $1(-)$ & Mult. & 0.98 & 1.02 \\
\hline Break loop pump speed multiplier & $1(-)$ & Mult. & 0.9 & 1.1 \\
\hline Accumulator pressure setpoint & $4.14(\mathrm{MPa})$ & Add. & -0.2 & 0.2 \\
\hline Accumulator liquid temperature & $334(\mathrm{~K})$ & Add. & -10 & 10 \\
\hline LPI mass flow rate & $88(\mathrm{~kg} / \mathrm{s})$ & Mult. & 0.95 & 1.05 \\
\hline Initial pressurizer pressure & $15.5(\mathrm{MPa})$ & Add. & -0.1 & 0.1 \\
\hline Initial pressurizer level & $8.8(\mathrm{~m})$ & Add. & -0.1 & 0.1 \\
\hline Initial total primary mass flow rate & $17357(\mathrm{~kg} / \mathrm{s})$ & Mult. & 0.96 & 1.04 \\
\hline Initial cold legs average temperature & $565(\mathrm{~K})$ & Add. & -2 & 2 \\
\hline \multicolumn{5}{|l|}{ Lognormal Distributed Parameters } \\
\hline Accumulator loss coeff. & $1(-)$ & Mult. & 0.5 & 2 \\
\hline Surge line coss coeff. & $1(-)$ & Mult. & 0.5 & 2 \\
\hline \multicolumn{5}{|l|}{ Uniform Distributed Parameters } \\
\hline Core loss coeff. & $0.51(-)$ & Mult. & 0.95 & 1.05 \\
\hline Bypass loss coeff. & $13.575(-)$ & Mult. & 0.95 & 1.05 \\
\hline Downcomer loss coeff. & $0.54(-)$ & Mult. & 0.95 & 1.05 \\
\hline Low core plate loss coeff. & $0.2(-)$ & Mult. & 0.95 & 1.05 \\
\hline Break cold leg loss Coeff. & $0.68(-)$ & Mult. & 0.95 & 1.05 \\
\hline Vessel liquid wall friction multiplier & $1(-)$ & Mult. & 0.95 & 1.05 \\
\hline Wall friction multiplier & $1(-)$ & Mult. & 0.95 & 1.05 \\
\hline Liquid choke flow coeff. & $1(-)$ & Mult. & 0.95 & 1.05 \\
\hline Vapour/two-phase choke flow coeff. & $1(-)$ & Mult. & 0.95 & 1.05 \\
\hline Gas-gap heat transfer Coeff. & $6300\left(\mathrm{~W} / \mathrm{m}^{2} \mathrm{~K}\right)$ & Mult. & 0.9 & 1.1 \\
\hline gap conductance coeff. & $1(-)$ & Mult. & 0.9 & 1.1 \\
\hline Clad thermal conductivity & $1(-)$ & Mult. & 0.9 & 1.1 \\
\hline Clad density & $6551.4\left(\mathrm{~kg} / \mathrm{m}^{3}\right)$ & Mult. & 0.95 & 1.05 \\
\hline Maximum containment pressure & $3.5 \mathrm{e} 5(\mathrm{~Pa})$ & Mult. & 0.85 & 1.15 \\
\hline Single phase liquid to wall heat transfer coeff. & $1(-)$ & Mult. & 0.9 & 1.1 \\
\hline Nucleate boiling heat transfer coeff. & $1(-)$ & Mult. & 0.9 & 1.1 \\
\hline Single phase vapor to wall heat transfer coeff. & $1(-)$ & Mult. & 0.9 & 1.1 \\
\hline Dispersed flow film boiling heat transfer coeff. & $1(-)$ & Mult. & 0.9 & 1.1 \\
\hline Film to transition boiling Tmin criterion & $1(-)$ & Mult. & 0.9 & 1.1 \\
\hline Transition boiling heat transfer coeff. & $1(-)$ & Mult. & 0.9 & 1.1 \\
\hline Critical Heat Flux (CHF) multiplier & $1(-)$ & Mult. & 0.8 & 1.2 \\
\hline Form loss coeff. & $1(-)$ & Mult. & 0.9 & 1.1 \\
\hline Interfacial drag (bubbly) coeff. & $1(-)$ & Mult. & 0.9 & 1.1 \\
\hline Interfacial drag (churn) coeff. & $1(-)$ & Mult. & 0.9 & 1.1 \\
\hline Interfacial drag (annular) coeff. & $1(-)$ & Mult. & 0.9 & 1.1 \\
\hline Interfacial drag (droplet) coeff. & $1(-)$ & Mult. & 0.9 & 1.1 \\
\hline
\end{tabular}




\begin{tabular}{l|c|c|c|c}
\hline $\begin{array}{l}\text { Interfacial drag (dispersed flow film boiling) } \\
\text { coeff. }\end{array}$ & $1(-)$ & Mult. & 0.9 & 1.1 \\
\hline Interfacial drag (inverted slug flow) coeff. & $1(-)$ & Mult. & 0.9 & 1.1 \\
\hline Interfacial drag (inverted annular flow) coeff. & $1(-)$ & Mult. & 0.9 & 1.1 \\
\hline Wallis c for counter current flow limitation & $0.8625(-)$ & Mult. & 0.9 & 1.1 \\
\hline Accumulator liquid volume & $10.564\left(\mathrm{~m}^{3}\right)$ & Add. & -0.5 & 0.5 \\
\hline Accumulator gas volume & $13.583\left(\mathrm{~m}^{3}\right)$ & Add. & -0.8 & 0.8 \\
\hline $\begin{array}{l}\text { Vapour to wall inverted annular heat transfer } \\
\text { coeff. }\end{array}$ & $1(-)$ & Mult. & 0.9 & 1.1 \\
\hline Liquid to wall inverted annular heat transfer coeff. & $1(-)$ & Mult. & 0.9 & 1.1 \\
\hline Initial upper head temperature & $570(\mathrm{~K})$ & Add. & 0 & 10 \\
\hline Liquid to interface bubbly-slug heat transfer coeff. & $1(-)$ & Mult. & 0.9 & 1.1 \\
\hline $\begin{array}{l}\text { Liquid to interface annular-mist heat transfer } \\
\text { coeff. }\end{array}$ & $1(-)$ & Mult. & 0.9 & 1.1 \\
\hline Liquid to interface transition heat transfer coeff. & $1(-)$ & Mult. & 0.9 & 1.1 \\
\hline Liquid to interface stratified heat transfer coeff. & $1(-)$ & Mult. & 0.9 & 1.1 \\
\hline $\begin{array}{l}\text { Vapour to interface bubbly-slug heat transfer } \\
\text { coeff. }\end{array}$ & $1(-)$ & Mult. & 0.9 & 1.1 \\
\hline $\begin{array}{l}\text { Vapour to interface annular-mist heat transfer } \\
\text { coeff. }\end{array}$ & $1(-)$ & Mult. & 0.9 & 1.1 \\
\hline Vapour to interface transition heat transfer coeff. & $1(-)$ & Mult. & 0.9 & 1.1 \\
\hline Vapour to interface stratified heat transfer coeff. & $1(-)$ & Mult. & 0.9 & 1.1 \\
\hline Sub-cooled boiling heat transfer coeff. & $1(-)$ & Mult. & 0.9 & 1.1 \\
\hline Departure from nucleate boiling/CHF & $1(-)$ & Mult. & 0.9 & 1.1 \\
\hline Fuel thermal conductivity before burst coeff. & $1(-)$ & Mult. & 0.9 & 1.1 \\
\hline Cladding metal-water reaction rate coeff. & $1(-)$ & Mult. & 0.9 & 1.1 \\
\hline Rod internal pressure coeff. & $1(-)$ & Mult. & 0.9 & 1.1 \\
\hline Burst temperature coeff. & $1(-)$ & Mult. & 0.9 & 1.1 \\
\hline Burst strain coeff. & $1(-)$ & Mult. & 0.9 & 1.1 \\
\hline & & & & \\
\hline
\end{tabular}

\subsection{Identification of relevant safety-related systems, trains, and components. Assumptions of systems configuration}

The safety-related systems involved in the accidental transient are the reactor protection system, accumulators, and low-pressure injection systems. There are four accumulators and four LPIS injections. Thus, when the break occurs reactor scram is produced and the reactor coolant pumps stop. The pressure inside the reactor coolant system rapidly falls and, when it reaches $4.14 \mathrm{MPa}$, the injection begins through the accumulators. The pressure cannot be recovered and when it reaches 1.42 MPa LPIS injection starts.

The conditions imposed for the thermal hydraulic transient simulation related to safety systems were extracted from the BEMUSE project [4] and are summarized as follows:

- No actuation of the high-pressure injection system (HPIS).

- Injection from accumulators at 4.14 MPa.

- Low-pressure injection system (LPIS) initiate at 1.42 MPa.

- Containment pressure imposed as a function of time after the break.

- Reactor coolant pumps velocity imposed as a function of time after the break.

- Power after scram imposed by means of a multiplier as a function of time after the break.

\subsection{TRACE model of the 4-loops PWR-Westinghouse}


Figure 2 outlines the primary system modeled for TRACE V5.0 Patch 4 using the SNAP suite, which includes a three-dimensional component type VESSEL, which represents the reactor pressure vessel including the core.

The reactor pressure vessel (RPV) consists of 31 axial, 5 radial and 8 azimuthal nodes. The tridimensional vessel permits represent peak clad temperature in a more realistic way. The core is composed of the cells comprised to 6 to 23 axial nodes, 1 to 3 radial nodes, and for all 8 azimuthal nodes.

It also includes the four cooling loops (PIPES, 4 SGs, PRZ and 4 PUMPS). The safety systems involved in the accidental sequence are also modeled. The accumulators are modeled using PIPE and VALVE components in each loop, e.g. PIPE-190 and VALVE 194 in loop one. The LPIS injections are simulated as boundary conditions using a FILL component in each loop, e.g. FIL-192 in loop1. No safety injections were modeled in the broken loop, as it is considered that the coolant injected is completely lost through the break. Finally, the break is simulated as a double guillotine using three VALVE (VALVE 505, VALVE 507 and VALVE 213) and two BREAK (BREAK 500 and BREAK 501) components in the cold leg of Loop 2.

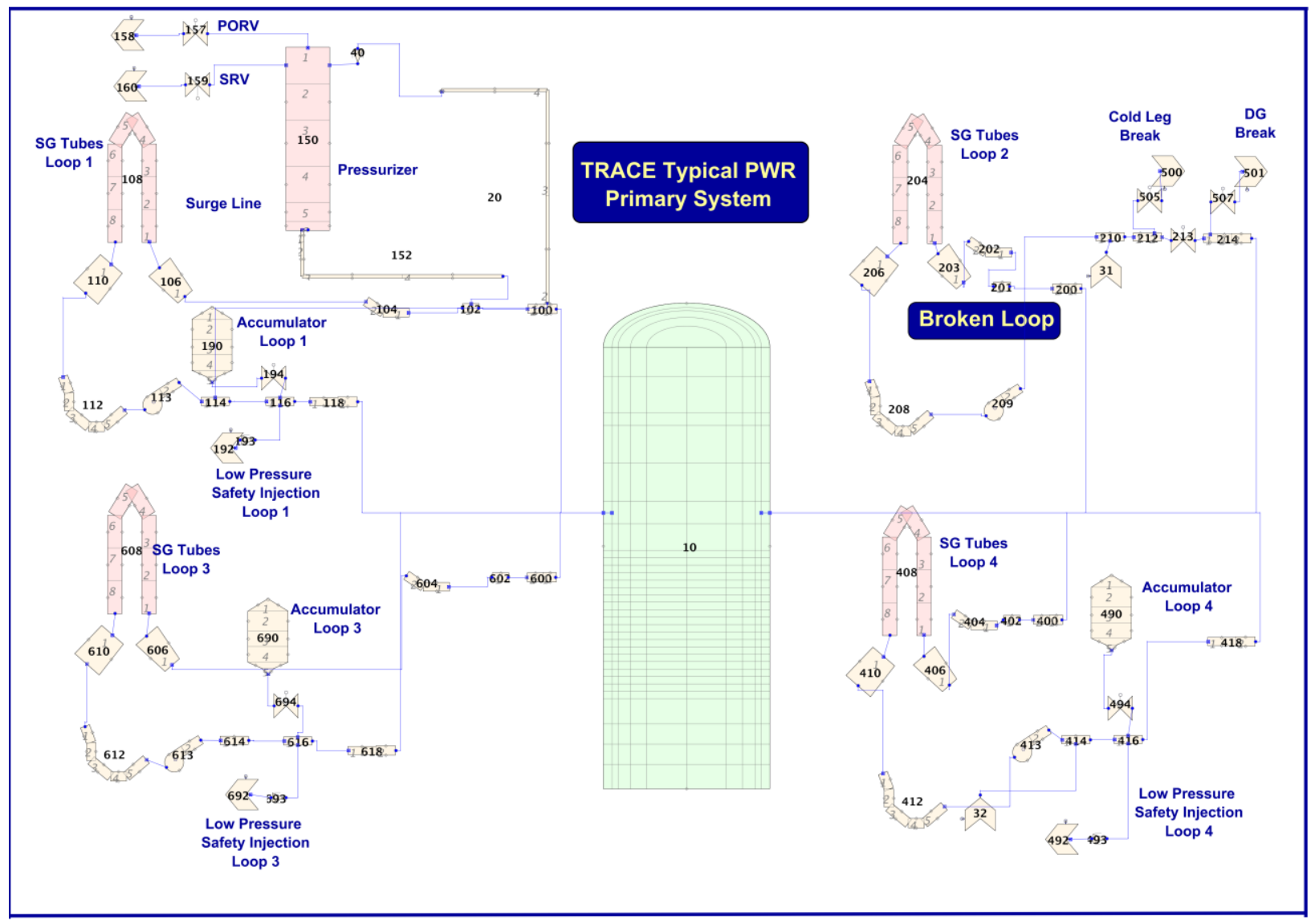

Figure 2. TRACE typical 4-loops PWR. Primary System SNAP view.

\section{RESULTS OF THE UNCERTAINTY ANALYSIS}

This section summarizes the results of the uncertainty analysis of the BEPU approach highlighted in Figure 1 (Section 2). The uncertainty analysis was carried out using the methods introduced in Section 3 and the model and data provided in Section 4. The results of the alternative methods are compared by means of the performance metrics proposed in Section 2. 


\subsection{PCT evolution for the base case}

The LBLOCA transient was simulated using the SNAP-TRACE code model with the input parameters at their nominal value, which results in the PCT evolution shown in Figure 3. This simulation of the Base Case (see Figure 1) reached a PCTmax of 1221.1 K.

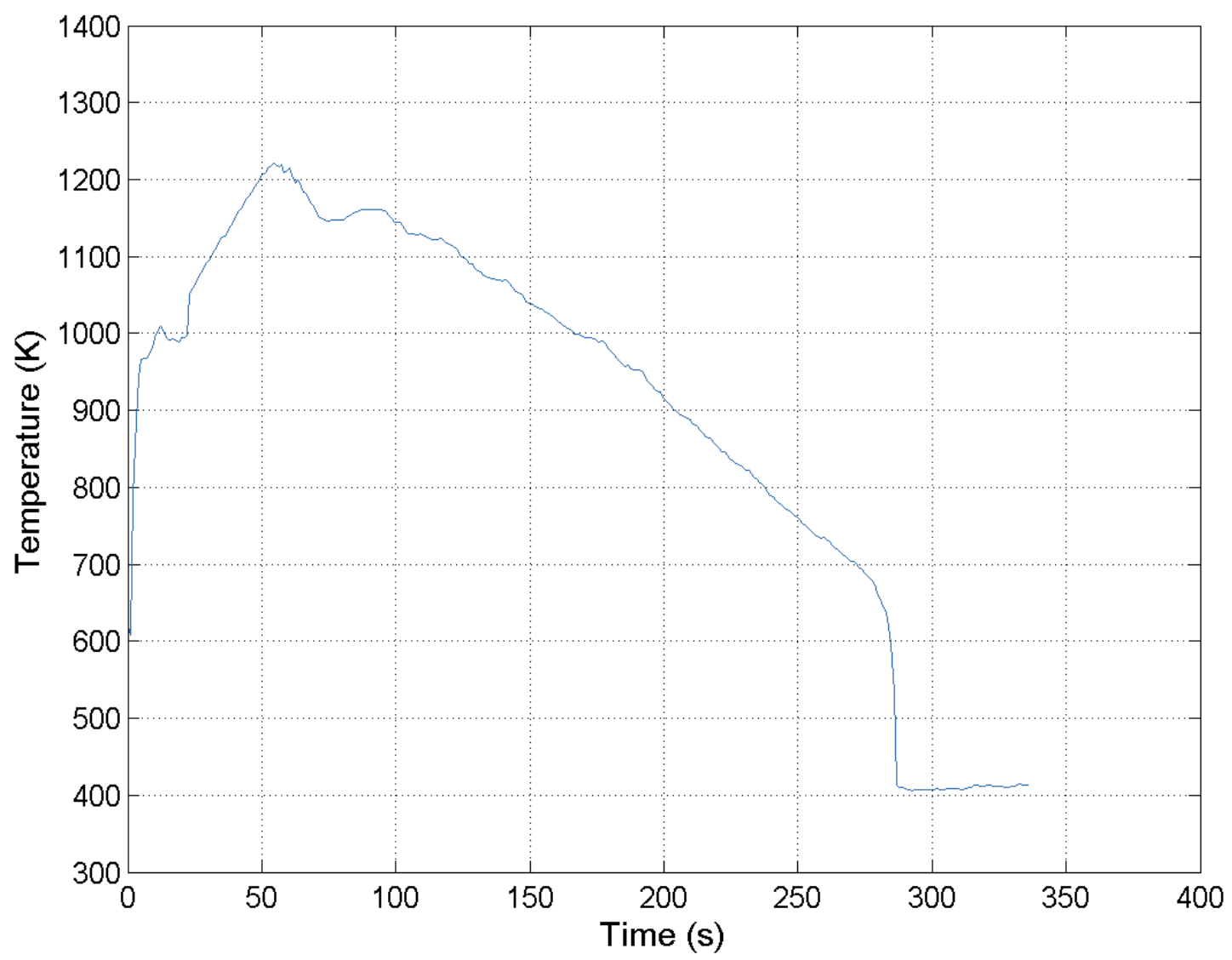

Figure 3. TRACE result for the PCT evolution with nominal input parameters

\subsection{5-percentile of the PCTmax as a reference value}

To obtain the 95-percentile of the PCTmax, $Z=1000$ simulations of the transient were performed using the SNAP-TRACE code, varying the nominal values of the parameters given in Table 3 according to their probability distributions and ranges of variation. After all the simulations, the set of PCT evolutions depicted in Figure 4 were obtained. It can be seen that the PCT evolutions have similar shapes and different widths and lengths. 


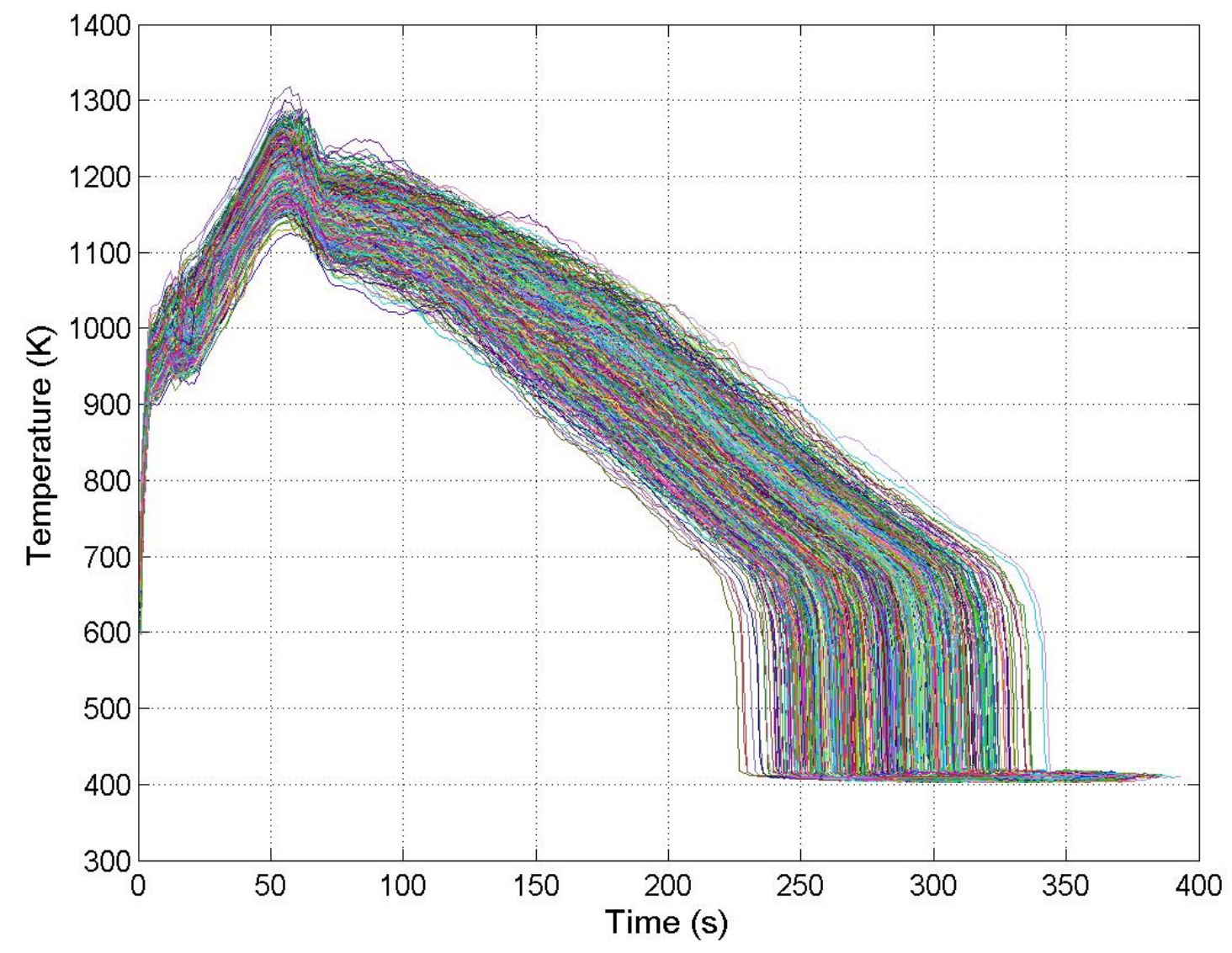

Figure 4. TRACE results for the PCT evolution of one thousand simulations

These simulations are intended to be used for the purpose of estimating the density function of the PCTmax empirically. Thus, Figure 5 shows the empirical probability density function $(p d f)$ and the histogram obtained for PCTmax from the 1000 simulations. In order to compute the performance metrics in the comparison of the different uncertainty analysis methods, the 95percentile of the $p d f$ is considered to be the $\mathrm{SV}_{\text {ref }}$ in Eq. (15), which has a value of $\mathrm{SV}_{\text {ref }}=1259.5$ $\mathrm{K}$. 


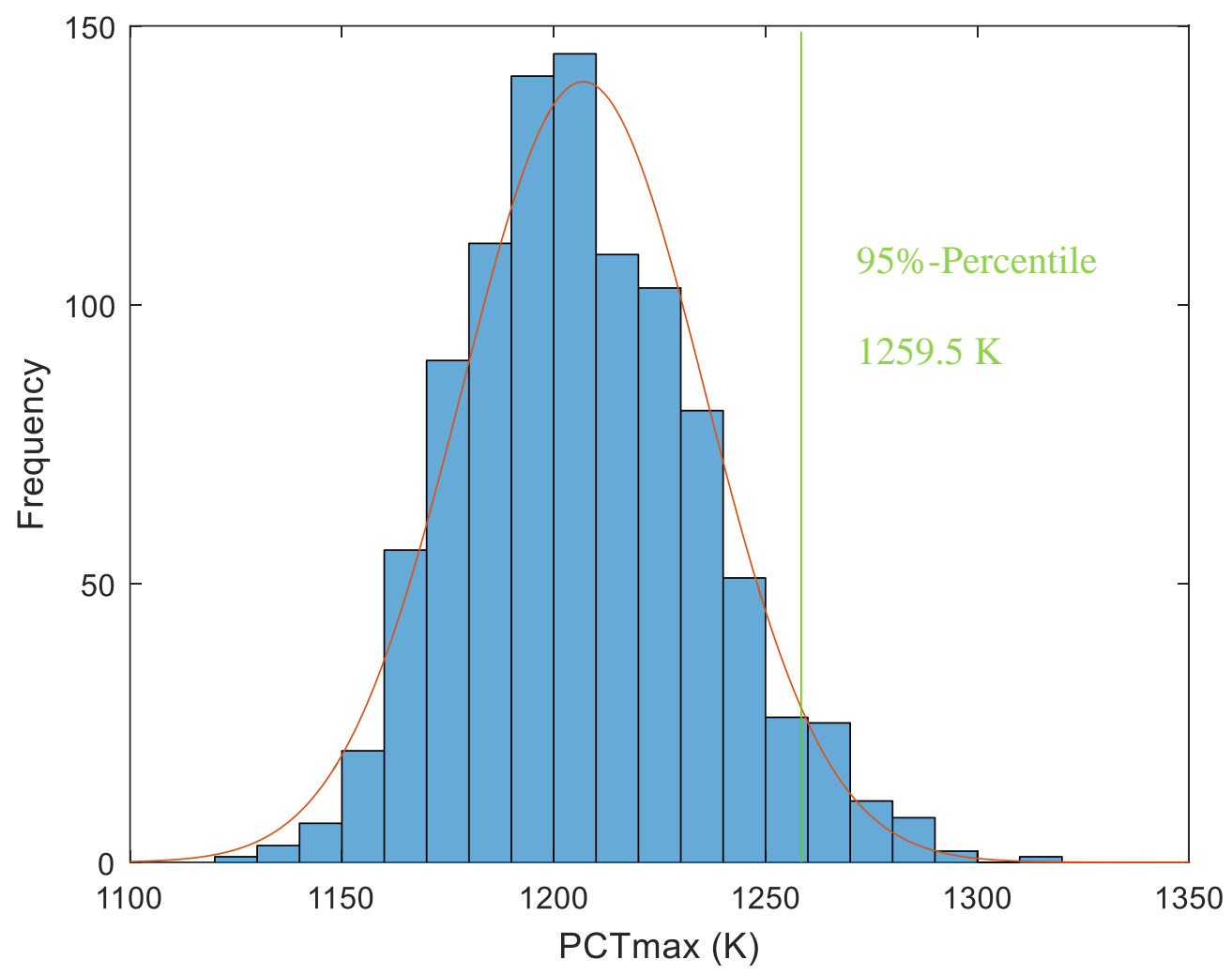

Figure 5. Histogram and probability density function (pdf) obtained from 1000 simulations of TRACE code for PCTmax.

\subsection{Uncertainty analysis: Wilks versus other non-parametric methods}

This section gives the results of the estimation of the PCTmax 95/95 TL by the different methods described in Section 3, i.e. Wilks (W), Beran and Hall (BH), Hutson (HU) and biascorrected accelerated bootstrap (BO) methods. The estimation was performed adopting the first and higher order statistics (2, 3 and 4 order), which correspond with sample sizes $n=\{59,93$, $124,153\}$, respectively.

In obtaining each particular PCTmax 95/95 TL (identified as TL in Section 3.5) a subset of $n$ samples of PCTmax values are randomly extracted from the $Z=1000$ simulations carried out and the $T L_{j}$ is determined for each non-parametric method from the fundamentals introduced in Sections 3.1 to 3.4. This process is repeated $N=999$ times, so that a sample of $T L_{\mathrm{j}}$ values is obtained, $j=1, N$, for each method, which permits the distribution of the PCTmax $\mathrm{TL}_{\mathrm{j}}$ to be estimated according to each method. This distribution will provide the performance measures for each method (see Section 5.4). This process is repeated for the different sizes of $n=\{59$, $93,124,153\}$.

Table 4 shows the mean, standard deviation, minimum and maximum value of the PCTmax TL estimation obtained using the Wilks, Beran-Hall, Huston and Bootstrapped methods for different samples sizes, $n$. Thus, the FOS $(n=59)$ with the Wilks' method results in a mean PCTmax TL value of $1280.97 \mathrm{~K}$. Also given is the dispersion of the PCTmax TL values among the different repetitions, which can be confirmed by the standard deviation of $14.62 \mathrm{~K}$. The alternative methods based on fractional order statistics provide results similar to Wilks for the 
same sample size $(n=59)$, since the mean values for Beran-Hall and Hutson are $1280.98 \mathrm{~K}$ and $1280.62 \mathrm{~K}$, respectively, and their dispersions are practically the same as Wilks', since BeranHall gives a standard deviation of $14.5 \mathrm{~K}$ and Hutson 14.38 K. The Bootstrap method gives a slightly lower mean value than the others $(1277.38 \mathrm{~K})$ and also lower dispersion, with a standard deviation of $13.29 \mathrm{~K}$.

Figure 6 represents the Box-Whisker plot of the PCTmax TL distribution for several $n$ values. The figure shows a positive asymmetry in the PCTmax TL distribution obtained using the different non-parametric methods with small sample sizes $(n=59)$. This asymmetry decreases as the sample size increases. It can be seen that the most accurate estimates are obtained by increasing the sample size. It also confirms the very conservative estimation of the Wilks method for a sample size of 59 .

For sample size $n=93$ the results were similar to those for $n=59$, with the lowest mean and standard deviation values for the Bootstrap method, with values of $1272.78 \mathrm{~K}$ and $8.7 \mathrm{~K}$. respectively. The other methods provide similar results, but slightly higher than the Bootstrap values.

For higher sample sizes, $(n=124$ and 153) the results tend to become similar for all the uncertainty analysis methods. The Hutson and Bootstrap results have lower dispersion than Wilks and Beran-Hall. For example, for $n=153$, Bootstrap has a lower standard deviation value $(6.19 \mathrm{~K})$ than Hutson $(6.53 \mathrm{~K})$, Beran-Hall $(6.67 \mathrm{~K})$ and Wilks $(7.28 \mathrm{~K})$. The mean values of all four methods are practically identical.

Table 4. PCTmax 95/95 TL estimation results (in K).

\begin{tabular}{lllll}
\hline Method & Mean & Std Dev & Minimum & Maximum \\
\hline Wilks $\mathrm{n}=59$ & 1280.97 & 14.62 & 1241.6 & 1318.9 \\
Wilks n=93 & 1274.06 & 9.13 & 1243.6 & 1300.0 \\
Wilks n=124 & 1271.78 & 7.71 & 1249.2 & 1294.7 \\
Wilks n=153 & 1271.33 & 7.28 & 1248.1 & 1294.7 \\
Beran-Hall $\mathrm{n}=59$ & 1280.98 & 14.50 & 1242.5 & 1318.7 \\
Beran-Hall $\mathrm{n}=93$ & 1273.88 & 9.10 & 1249.6 & 1300 \\
Beran-Hall $\mathrm{n}=124$ & 1271.28 & 7.27 & 1249.4 & 1294.7 \\
Beran-Hall $\mathrm{n}=153$ & 1270.30 & 6.67 & 1250.0 & 1287.6 \\
Hutson $\mathrm{n}=59$ & 1280.62 & 14.38 & 1241.1 & 1318.6 \\
Hutson $\mathrm{n}=93$ & 1274.19 & 9.19 & 1242.6 & 1299.6 \\
Hutson $\mathrm{n}=124$ & 1271.24 & 7.23 & 1249.4 & 1294.6 \\
Hutson $\mathrm{n}=153$ & 1270.36 & 6.53 & 1255.4 & 1288.2 \\
Bootstrap $\mathrm{n}=59$ & 1277.38 & 13.29 & 1241.9 & 1318.9 \\
Bootstrap $\mathrm{n}=93$ & 1272.78 & 8.70 & 1246.2 & 1299.2 \\
Bootstrap $\mathrm{n}=124$ & 1271.04 & 7.26 & 1248.1 & 1291.8 \\
Bootstrap n=153 & 1269.42 & 6.19 & 1248.5 & 1288.0 \\
\hline
\end{tabular}




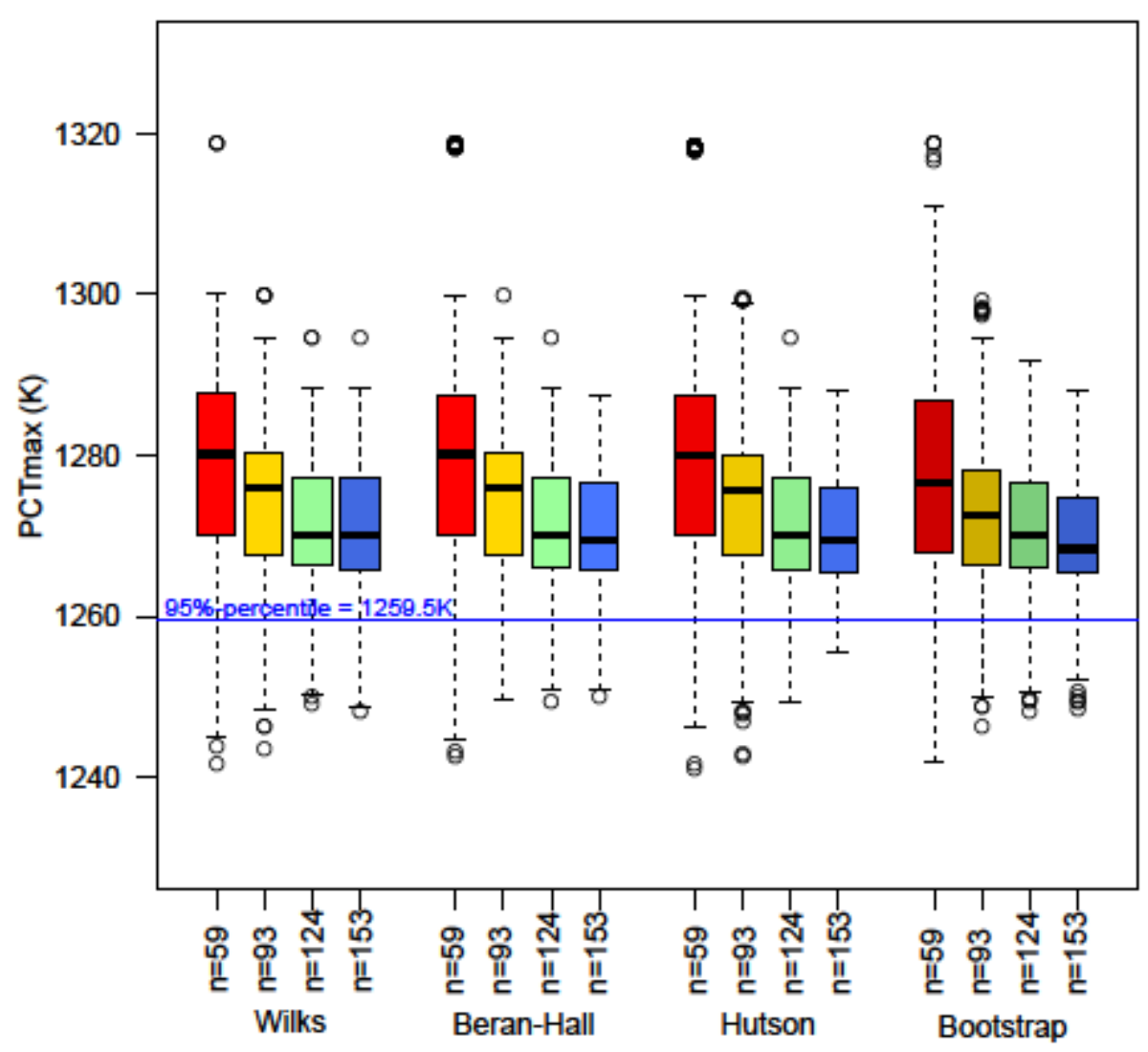

Figure 6. Box-Whisker plot for PCTmax (in K) 95/95 TL using different uncertainty analysis methods

\subsection{Performance metrics}

The PCTmax 95/95 TL distributions of each non-parametric method given above are used here to obtain the performance measures for each method following the procedure introduced in Section 3.5. This procedure is carried out for several sizes of $n=\{59,93,124,153\}$ to permit the comparison of the results provided by the four uncertainty methods according to the order statistic adopted.

Table 5 shows the coverage mean (CM), standard deviation coverage (CD), the coefficient of variation $(\mathrm{CV})$ and conservativeness (CC) estimated for the Wilks, Beran-Hall, Hutson and bootstrap methods for different sample sizes. The results in this table show that the four methods provide good CM values of around 97-98\%. In terms of CD, which measures dispersion, the values obtained by all methods are also quite similar, but for higher $n$ sizes ( $n=124$ and 153) the Bootstrap and Hutson methods gave better results than Beran-Hall and Wilks. However, for the lowest $n$ sample size $(n=59)$ the Bootstrap method had the worst result in terms of dispersion, i.e. CD value. The Wilks' method obtained the worst results in terms of dispersion for $n=124$ and $n=153$. However, Beran-Hall and Wilks are more robust than Bootstrap and Hutson for lower $n$ sizes $(59,93)$, based on the CV metric, while the robustness of the Bootstrap and Hutson methods is better than the Beran-Hall and Wilks methods for higher $n$ sizes $(124,153)$, based on the same CV metric. Regarding the CC metric, Hutson and Bootstrap provide values no higher than $95 \%$ for $n=59$ and $n=93$, while Wilks and Beran-Hall provide CC values higher than $95 \%$ for all the $n$ sample sizes. In general, both the Beran and Hall and the Wilks' methods are conservative for any sample size $n$, the former being less conservative than Wilks. 
Table 5. Performance metrics for the PCTmax 95/95 TL estimation

\begin{tabular}{lllll}
\hline Method & $\begin{array}{l}\text { Coverage } \\
\text { Mean }(\mathbf{C M})\end{array}$ & $\begin{array}{l}\text { Coverage } \\
\text { Std Dev }(\mathbf{C D})\end{array}$ & $\begin{array}{l}\text { Coeff. of } \\
\text { Var. }(\mathbf{C V})\end{array}$ & $\begin{array}{l}\text { Conservative- } \\
\text { ness }(\mathbf{C C})\end{array}$ \\
\hline Wilks n=59 & 98.47 & 1.55 & 1.57 & 96.6 \\
Wilks n=93 & 97.94 & 1.42 & 1.45 & 96.4 \\
Wilks n=124 & 97.67 & 1.32 & 1.35 & 96.2 \\
Wilks n=153 & 97.62 & 1.30 & 1.33 & 96.5 \\
Beran-Hall $\mathrm{n}=59$ & 98.43 & 1.55 & 1.57 & 95.7 \\
Beran-Hall n=93 & 97.91 & 1.43 & 1.46 & 95.3 \\
Beran-Hall $\mathrm{n}=124$ & 97.61 & 1.26 & 1.29 & 96.3 \\
Beran-Hall $\mathrm{n}=153$ & 97.47 & 1.23 & 1.27 & 95.3 \\
Hutson $\mathrm{n}=59$ & 98.37 & 1.59 & 1.62 & 95.0 \\
Hutson $\mathrm{n}=93$ & 97.89 & 1.50 & 1.53 & 94.6 \\
Hutson $\mathrm{n}=124$ & 97.57 & 1.25 & 1.29 & 96.2 \\
Hutson $\mathrm{n}=153$ & 97.47 & 1.16 & 1.19 & 96.5 \\
Bootstrap n=59 & 98.10 & 1.70 & 1.73 & 93.0 \\
Bootstrap n=93 & 97.73 & 1.41 & 1.45 & 94.6 \\
Bootstrap n=124 & 97.58 & 1.30 & 1.34 & 95.2 \\
Bootstrap n=153 & 97.32 & 1.17 & 1.20 & 95.6 \\
\hline
\end{tabular}

\section{CONCLUDING REMARKS}

The main advantage of using FOS based on Wilks' formulae is that it usually provides a conservative result with a small number of computer code runs. Although the Wilks' method is mainly used in this kind of analysis, other non-parametric methods could also be used to estimate the 95/95 TL. The study presented in this paper compares the use and performance of alternative non-parametric methods in obtaining FOM tolerance intervals within a BEPU framework, based on $1^{\text {st }}$ to $4^{\text {th }}$ order statistics. Three methods are investigated, i.e. Hutson and Beran-Hall methods in the context of fractional statistics and a Bootstrap method. These methods are applied to estimate the PCTmax 95/95 TL within the uncertainty analysis of a Large-Break Loss of Coolant Accident in the cold leg of a Pressurized Water Reactor, using the thermal-hydraulic code TRACE to perform the simulations. As expected, in all the methods the higher $\left(2^{\text {nd }}\right.$ to $\left.4^{\text {th }}\right)$ order statistics usually produced less conservative and more accurate results at the expense of additional code runs.

Four performance metrics were proposed to evaluate the goodness of the results. From the case study it was found that for lower $n$ sizes $(n=59$ and 93) the Bootstrap method provided the best results in terms of coverage mean (CM). However, it had the largest coverage standard deviation (CD) values, which gave the worst scores and thus affected robustness (CV). Bootstrap also provided conservativeness (CC) values lower than $95 \%$. According to the performance metrics obtained for these sizes, the best results were thus found by the BeranHall and Wilks methods, which provided similar results for robustness and both obtained very good CC values above 95\%. However, for higher $n$ sizes $(n=124$ and 153), the Bootstrap method provided the best results. The Beran-Hall method gave very similar results to those obtained by the Bootstrap method for $n=124$. Thus, in general, the performance of the methods improves with sample size as all four methods performed in a very similar way for the largest $n$ sizes. The main differences were found for the lowest $n$ sizes, with which the Beran-Hall method performed best. Even so, with the exception of the Bootstrap method, one could conclude that there are no significant differences between the methods. 
This kind of analysis should be extended to other transients considered important in nuclear safety, in which the figures of merit may change from the one proposed in this paper. Moreover, it could be interesting to analyze if the consideration of one figure of merit is sufficient to quantify the transient uncertainty or more variables should be considered in the uncertainty analysis.

\section{ACKNOWLEDGEMENTS}

This work was carried out partially with the support of the Programa de Apoyo a la Investigación y Desarrollo of the Universitat Politècnica de València (PAID UPV).

The authors are also grateful to the Spanish Consejo de Seguridad Nuclear for the financial support they provided for Research Projects SIN/4078/2013/640 (MASA Project) and STN/4524/2015/640 (CAMP Project).

\section{REFERENCES}

[1] IAEA, 2009. Deterministic Safety Analysis for Nuclear Power Plants. IAEA Specific Safety Guide No. SSG-2. International Atomic Energy Agency, Vienna.

[2] IAEA, 2002. Accident Analysis for Nuclear Power Plants. Safety Reports Series No. 23. International Atomic Energy Agency, Vienna.

[3] IAEA, 2008. Best Estimate Safety Analysis for Nuclear Power Plants: Uncertainty Evaluation. Safety Reports Series Nº. 52. International Atomic Energy Agency, Vienna.

[4] Prošek A., Mavko B., 2003. Review of Best Estimate Plus Uncertainty Methods of Thermal Hydraulic Safety Analysis. In: Proceedings of the International Conference Nuclear Energy for Central Europe, Portorož, Slovenia.

[5] Nutt W. T., Wallis G. B., 2003. Evaluation of nuclear safety from the outputs of computer codes in the presence of uncertainties. 2003. Reliability Engineering and System Safety. 83, 57-77.

[6] Pourgol-Mohammad, M., 2009. Thermal-Hydraulics system codes uncertainty assessment: A review of the methodologies. Annals of Nuclear Energy: 36, 1774-1786

[7] Wilson G. E., 2013. Historical insights in the development of Best Estimate Plus Uncertainty safety analysis. Annals of Nuclear Energy. 52, 2-9.

[8] Perez et al., 2011. Uncertainty and sensitivity analysis of a LBLOCA in a PWR Nuclear Power Plant: Results of the Phase V of the BEMUSE program. Nuclear Engineering and Design. 241(10), 4206-4222.

[9] Glaeser H., Hofer E., Kloos M., Skorek T., 1994 Uncertainty and sensitivity analysis of a post-experiment calculation in thermal hydraulics. Reliability Engineering and System Safety. 45, 19-33. 
[10] Zio E., Di Maio F., Tong J., 2010. Safety margins confidence estimation for a passive residual heat removal system. Reliability Engineering and System Safety. 95, 828-836.

[11] Gabraskas D., Nakayama M. K., Denning, R., Aldemir T., 2016. Advantages of variance reduction techniques in establishing confidence intervals for quantiles.

[12] Pourgol-Mohammad, M., 2007. Integrated Methodology for Thermal-Hydraulics Uncertainty Analysis (IMTHUA). PhD dissertation. University of Maryland.

[13] D'Auria, F., Camargo, C., Mazzantini, O., 2012. The Best Estimate Plus Uncertainty (BEPU) approach in licensing of current nuclear reactors. Nuclear Engineering and Design. 248, 317-328.

[14] Martorell, S., Martorell, P.; Martón, I.; Sánchez, A.I., Carlos S., 2017. Reliability Engineering and System Safety. 160, 136-150.

[15] Applied Programming Technology, Inc., 2012. Symbolic Nuclear Analysis Package (SNAP). User's Manual. Version 2.2.1. Applied Programming Technology, Inc., Bloomsburg

[16] USNRC. 2014a. TRACE V5.840 Theory Manual Field Equations, Solution Methods, and Physical Models. U. S. Nuclear Regulatory Commission, Washington, DC.

[17] USNRC. 2014b. TRACE V5.840 User's Manual Volumes 1 and 2: Modeling Guidelines. U. S. Nuclear Regulatory Commission, Washington, DC.

[18] ]USNRC, 1989a. Regulatory Guidance 1.157. Best-Estimate Calculations of Emergency Core Cooling System Performance. U. S. Nuclear Regulatory Commission, Washington, DC.

[19] Martin, R.P, O’Dell, L.D., 2005. AREVA's realistic large break LOCA analysis methodology. Nuclear Engineering and Design, 235, 1713-1725.

[20] Wilks, S.S., 1941. Determination of Sample Sizes for Setting Tolerance Limits. The Annals of Mathematical Statistics. 12, 91-96.

[21] Wilks, S.S., 1942. Statistical prediction with special reference to the problem of tolerance limits. The Annals of Mathematical Statistics. 13(4), 400-409.

[22] Beran, R., Hall, P., 1993. Interpolated nonparametric prediction intervals and confidence interval. J. Royal Statistic Soc. (B) 55, 643-652.

[23] Hutson, A.D., 1999. Calculating nonparametric confidence intervals for quantiles using fractional order statistics. Journal of applied statistics, 26(3), 343-353.

[24] Zio, E., Di Maio. F., 2008. Bootstrap and Order Statistics for Quantifying ThermalHydraulic Code Uncertainties in the Estimation of Safety Margins. Science and Technology of Nuclear Installations. 2008, Article ID340164. 
[25] Briggs, L.L., 2008. Uncertainty Quantification Approaches for Advanced Reactor Analyses. Nuclear Engineering Division, Argonne National Laboratory.

[26] Di Maio, F., Bandini, A., Zio, E., Carlos, S., Sanchez-Saez, F., Martorell, S., 2016. Bootstrapped-ensemble-based Sensitivity Analysis of a trace thermal-hydraulic model based on a limited number of PWR large break loca simulations. Reliab. Eng. Syst. Safe. 153, 122-134. http://doi.org/10.1016/j.ress.2016.04.013.

[27] Stigler, M.S., 1977. Fractional Order Statistics, with Applications. Journal of the American Statistical Association. 72 (359), 544-550.

[28] Efron, B., Tibshirani, R., 1986. Bootstrap methods for standard errors, confidence intervals, and other measures of statistical accuracy. Statistical Science 1 (1), 54-77.

[29] Di Ciccio, T. J., Efron, B., 1996. Bootstrap Confidence Intervals. Statistical Science 11(3), 189-228.

[30] C. Z. Mooney and R. D. Duval, Bootstrapping: A Nonparametric Approach to Statistical Inference, Quantitative Applications in the Social Sciences, Sage, Newburry Park, CA, USA, 1993.

[31] Picheny, V., Kim, N., Haftka, T., Queipo, N.V., 2008. Conservative Predictions Using Surrogate Modeling. In: Proceedings of the 49th AIAA/ASME/ASCE/AHS/ASC Structures, Structural Dynamics, and Materials, Schaumburg, IL.

[32] USNRC, 2015. 10 CFR 50.46. Acceptance criteria for emergency core cooling systems for light-water nuclear power reactors. U. S. Nuclear Regulatory Commission, Washington, DC.

[33] USNRC, 1989b, Quantifying Safety Margins: Application of Code Scaling, Applicability, and Uncertainty Evaluation Methodology to a Large -Break Loss-ofCoolant Accident. NUREG/CR-5249, EGG-2659. U. S. Nuclear Regulatory Commission, Washington, DC.

[34] Young, M.Y., Bajorek S.M., Nissley, M.E., Hochreiter, L.E., 1998. Application of code scaling applicability and uncertainty methodology to the large break loss of coolant. Nuclear Engineering and Design. 186, 39-52.

[35] NEA/CSNI/R, 2016. PREMIUM, a benchmark on the quantification of the uncertainty of the physical models in the system thermal-hydraulic codes: methodologies and data review. 